\title{
ON A RECTANGLE INCLUSION PROBLEM
}

\author{
JANUSZ PAWLIKOWSKI
}

(Communicated by Andreas R. Blass)

\begin{abstract}
We show that if every set of reals of size $2^{\aleph_{0}}$ contains a meager-toone continuous image of a set that cannot be covered by less than $2^{\aleph_{0}}$ meager sets, then there exists a null (Lebesgue measure zero) subset of the plane $\mathbb{R} \times \mathbb{R}$ that meets every nonnull rectangle $X \times Y$. The antecedent is satisfied, e.g., if $\omega_{2}$ Cohen reals are added to a model of the continuum hypothesis.
\end{abstract}

Martin's Axiom implies that a conull (i.e., with null complement) subset of the Euclidean plane $\mathbb{R} \times \mathbb{R}$ contains a nonnull rectangle $X \times Y$. Fremlin [5], Problem AS (see also [6], 3K), asked if this is true in ZFC.

It is known that there exists a conull subset of $\mathbb{R} \times \mathbb{R}$ which contains no rectangle $X \times Y$ with one side nonnull and the other measurable and nonnull. Namely, let $E=\{\langle x, y\rangle \in \mathbb{R} \times \mathbb{R}: x+y \in F\}$, where $F=\mathbb{R} \backslash \mathbb{Q}$. Clearly, $E$ is a conull subset of $\mathbb{R} \times \mathbb{R}$. If $X \times Y \subseteq E$, then $X+Y \subseteq F$. But, by a theorem of Steinhaus (see [9]), if $X$ is measurable nonnull and $Y$ is nonnull, then $X+Y$ has nonempty interior; hence $X+Y$ cannot be contained in $F$. (However, Brodskij and Eggleston (see [4]) showed that a measurable nonnull subset of $\mathbb{R} \times \mathbb{R}$ always contains a rectangle $X \times Y$ with $X$ perfect and $Y$ measurable nonnull.)

Consider the following proposition.

(+) If an $\dot{\mathbf{F}}_{\sigma}$ subset of $\mathbb{R} \times \mathbb{R}$ contains a nonnull rectangle $X \times Y$, then it contains a measurable nonnull rectangle $A \times B$.

Proposition (+) implies that there exists a conull subset of $\mathbb{R} \times \mathbb{R}$ which contains no nonnull rectangle. Any conull $F_{\sigma}$ subset of the set $E$ considered above will do.

Proposition $(+)$ has other interesting consequences (see [1]). For instance, it follows from ( + ) that if $X$ and $Y$ are nonnull subsets of $\mathbb{R}$, then $X+Y$ is nonmeager, hence every meager subgroup of $\mathbb{R}$ is null. (If $X+Y$ is covered by an $\mathbf{F}_{\sigma}$ set $F$, then $F^{*}=\{(x, y): x+y \in F\}$ is an $\mathbf{F}_{\sigma}$ cover for $X \times Y$. By $(+), F^{*}$ contains a closed nonnull rectangle $A \times B$. By the theorem of Steinhaus mentioned above, $A+B$ has nonempty interior. Hence, $F$ has nonempty interior.)

Received by the editors January 24, 1994 and, in revised form, February 25, 1994.

1991 Mathematics Subject Classification. Primary 03E05, 03E15, 28A05, 54A35.

Key words and phrases. Subsets of the plane, nonnull rectangles, closed null sets.

Supported by KBN grant PB 210179101 . 
Consistency of $(+)$ was shown independently by Friedman and Shelah (see [1] and [2]). The model used was $\omega_{2}$ Cohen reals over a model of the continuum hypothesis.

In this paper we show that $(+)$ is implied by a combinatorial condition whose consistency has been known for some time.

Denote by $\mathscr{P}(\mathbb{R})$ the family of all subsets of $\mathbb{R}$. Let $\lambda$ be a cardinal number. For $\mathscr{A} \subseteq \mathscr{P}(\mathbb{R})$ and $X \subseteq \mathbb{R}$ write $X \in \mathscr{A}_{\lambda}$ if $X$ can be covered by less than $\lambda$ sets from $\mathscr{A}$, otherwise write $X \in \mathscr{A}_{\lambda}^{+}$. Write also $X \in \mathscr{A}^{+}$if $X \in \mathscr{A}_{2}^{+}$.

Let $\mathscr{M}$ be the $\sigma$-ideal of meager subsets of $\mathbb{R}$. Say that a function is meagerto-one if preimages of points are meager.

For $D \subseteq \mathbb{R} \times \mathbb{R}$ and $y \in \mathbb{R}$ let $D^{y}$ denote the horizontal section of $D$ determined by $y$, i.e., $D^{y}=\{x \in \mathbb{R}:\langle x, y\rangle \in D\}$.

Consider the following condition.

$(*)_{\lambda}$ Every set of reals of size $\lambda$ contains a meager-to-one continuous image of a set from $\mathscr{M}_{\lambda}^{+}$.

Note that we do not weaken $(*)_{\lambda}$ if we replace 'continuous' by 'Baire measurable'. This is because Baire measurable functions are continuous on comeager sets (see [9]).

It is folklore that $(*)_{2^{\aleph_{0}}}$ holds if $\omega_{2}$ Cohen reals are added to a model of the continuum hypothesis. For example, Miller [8], p. 577, showed that in this model every set of reals of size $2^{\aleph_{0}}$ contains a one-to-one continuous image of a $\left(2^{\aleph_{0}}, \aleph_{1}\right)$-Lusin set. $(X \subseteq \mathbb{R}$ is a $(\lambda, \kappa)$-Lusin set if $|X|=\lambda$ and $|X \cap S|<\kappa$ for all meager $S$.) This immediately gives $(*)_{2^{\aleph_{0}}}$.

We prove:

Theorem. $(*)_{2^{\aleph_{0}}} \Rightarrow(+)$.

The Theorem is a consequence of the following Lemma.

Lemma. Assume $(*)_{2^{\aleph_{0}}}$. Then, with every nonnull set $X \subseteq \mathbb{R}$ we can associate a family $\left\{E_{n}: n<\omega\right\}$ of closed nonnull subsets of $\mathbb{R}$ such that if $\dot{X} \subseteq \bigcup_{m<\omega} D_{m}$, $D_{m} \subseteq \mathbb{R}$ closed sets, then for some $m$ and $n, E_{n} \subseteq D_{m}$.

Proof of the Theorem. Suppose that $X \times Y \subseteq \mathbb{R} \times \mathbb{R}$ is a nonnull rectangle covered by $\bigcup_{m<\omega} F_{m}, F_{m} \subseteq \mathbb{R} \times \mathbb{R}$ closed. Clearly $X$ and $Y$ are nonnull. Let the $E_{n}$ 's be as in the Lemma. Let $G_{n m}=\left\{y: E_{n} \subseteq\left(F_{m}\right)^{y}\right\}$. Then $E_{n} \times G_{n m} \subseteq F_{m}$. Each $G_{n m}$ is closed. By the Lemma, the $G_{n m}$ 's cover $Y$. Indeed, let $y \in Y$. We have $X \times\{y\} \subseteq \bigcup_{m} F_{m}$. So, $X \subseteq \bigcup_{m}\left(F_{m}\right)^{y}$; hence, by the Lemma, some $\left(F_{m}\right)^{y}$ contains some $E_{n}$, i.e. $y \in G_{n m}$.

Since $Y$ is nonnull, it follows that some $G_{n m}$ is nonnull. Thus, we can take $E_{n} \times G_{n m}$ as our measurable nonnull rectangle, which is contained in $F_{m}$.

Proof of the Lemma. Assume $(*)_{2^{N_{0}}}$. Fix a nonnull set $X \subseteq \mathbb{R}$.

Claim. There is $Y \in \mathscr{H}_{2^{\alpha_{0}}}^{+}$and a meager-to-one continuous function $f: Y \mapsto X$ such that for each closed null set $W, f^{-1}[W] \in \mathscr{M}_{2^{\aleph_{0}}}$.

Proof. Let $\left\{E_{\xi}: \xi<2^{\aleph_{0}}\right\}$ be an enumeration of all closed null sets. Pick inductively $x_{\xi} \in X \backslash\left(\left\{x_{\zeta}: \zeta<\xi\right\} \cup \bigcup_{\zeta<\xi} E_{\zeta}\right) \quad\left(\xi<2^{\aleph_{0}}\right)$. This is possible because $X$ is not null and $\left\{x_{\zeta}: \zeta<\xi\right\} \cup \bigcup_{\zeta<\xi} E_{\zeta}$ is null. (By $(*)_{2^{\aleph_{0}}}, \mathbb{R} \in \mathscr{M}_{2^{\aleph_{0}}}^{+}$; by [7], Thm. 2.1, this implies that a union of less than $2^{\aleph_{0}}$ closed null sets is 
null.) By the construction, the $x_{\xi}$ 's are distinct and for every closed null set $W,\left|W \cap\left\{x_{\xi}: \xi<2^{\aleph_{0}}\right\}\right|<2^{\aleph_{0}}$.

By $(*)_{2^{\aleph_{0}}}$ there is $Y \in \mathscr{M}_{2^{\aleph_{0}}}^{+}$and a meager-to-one continuous function $f$ : $Y \mapsto\left\{x_{\xi}: \xi<2^{\aleph_{0}}\right\}$. Clearly $f^{-1}[W \cap X] \in \mathscr{M}_{2^{\aleph_{0}}}$, for each closed null $W$.

Let $\mathscr{U}$ be a countable base for $\mathbb{R}$. Let $\left\{U_{n}: n<\omega\right\}$ be an enumeration of all $U \in \mathscr{U}$ with the property that $\overline{f[U \cap Y]}$ is not null. Let $E_{n}=\overline{f\left[U_{n} \cap Y\right]}$ $(n<\omega)$.

To see that this works suppose that $X \subseteq \bigcup_{m<\omega} D_{m}, D_{m} \subseteq \mathbb{R}$ closed sets. Then $f^{-1}\left[D_{m} \cap X\right]$ 's are relatively closed in $Y$ sets that cover $Y$.

Suppose that for each $m$ and $U \in \mathscr{U}$ with $U \cap Y \subseteq f^{-1}\left[D_{m} \cap X\right], \overline{f[U \cap Y]}$ is null. Then, by the claim, $U \cap Y \in \mathscr{M}_{2^{\aleph_{0}}}$. It follows that $Y$ is a union of countably many sets from $\mathscr{M}_{2^{\aleph_{0}}}$ and countably many nowheredense sets. Hence $Y \in \mathscr{M}_{2^{\aleph_{0}}}$, which is a contradiction.

Thus, for some $U \in \mathscr{U}$ with $U \cap Y \subseteq f^{-1}\left[D_{m} \cap X\right], \overline{f[U \cap Y]}$ is not null, so it must be one of the $E_{n}$ 's. Clearly, $f[U \cap Y] \subseteq D_{m}$.

We shall now generalize the Lemma and the Theorem. Fix cardinals $\kappa \leq$ $\lambda \leq 2^{\aleph_{0}}, \operatorname{cf}(\kappa)>\omega$, and an arbitrary family $\mathscr{F} \subseteq \mathscr{P}(\mathbb{R})$ of closed sets. Note that $\mathscr{F}_{\kappa}^{+}$is a $\sigma$-ideal.

Definition. Let $X \subseteq \mathbb{R}$. Say that a family $\left\{F_{n}: n<\omega\right\}$ of closed sets is $\kappa$ dense (for $X$ ) if, whenever $X$ is covered by less than $\kappa$ closed sets, then some one of these closed sets covers some $F_{n}$. Say $\sigma$-dense for $\aleph_{1}$-dense.

Note. Let $\mathscr{I} \subseteq \mathscr{P}(\mathbb{R})$ be a $\sigma$-ideal. Every closed set from $\mathscr{I}^{+}$(so also every superset of such a set) has a $\sigma$-dense family contained in $\mathscr{I}^{+}$. Indeed, suppose that $X \in \mathscr{I}^{+}$is closed. Let $\mathscr{U}$ be a countable base for $\mathbb{R}$. Let $X^{*}=X \backslash \bigcup\{U \in$ $\mathscr{U}: U \cap X \in \mathscr{I}\}$. Then $X^{*}$ is closed and each $U \cap X^{*}(U \in \mathscr{U})$ is either empty or belongs to $\mathcal{I}^{+}$. As a $\sigma$-dense family we can just take the collection of those sets $\overline{U \cap X^{*}}(U \in \mathscr{U})$ which belong to $\mathscr{I}^{+}$. If $X^{*} \subseteq \bigcup_{m<\omega} D_{m}$, $D_{m} \subseteq \mathbb{R}$ closed, then for some $m, X^{*} \cap D_{m}$ has nonempty interior relatively to $X^{*}$ (Baire's category theorem). So, for some $U \in \mathscr{U}, U \cap X^{*} \neq \varnothing$ and $U \cap X^{*} \subseteq D_{m}$. Then $\overline{U \cap X^{*}} \subseteq D_{m}$, and, by the definition of $X^{*}, U \cap X^{*} \in$ $\mathcal{I}^{+}$.

It also follows that if $X$ is arbitrary and we can find a family of closed sets $\left\{F_{n}: n<\omega\right\} \subseteq \mathcal{I}^{+}$such that every $\mathbf{F}_{\sigma}$ set covering $X$ contains some $F_{n}$, then $X$ has a $\sigma$-dense family contained in $\mathscr{I}^{+}$.

Lemma 1. Let $\left\{F_{n}: n<\omega\right\}$ be a $\kappa$-dense family for $X$. Suppose that $X \times Y \subseteq$ $\bigcup_{\xi<\mu} D_{\xi}$, where $\mu<\kappa$ and $D_{\xi} \subseteq \mathbb{R} \times \mathbb{R}(\xi<\mu)$ are closed. Then there are $Y_{n \xi} \subseteq Y \quad(n<\omega, \xi<\mu)$ such that $\bigcup_{n, \xi} Y_{n \xi}=Y$ and for all $n$ and $\xi$, $F_{n} \times Y_{n \xi} \subseteq D_{\xi}$.

Proof. Let $Y_{n \xi}=\left\{y \in Y: F_{n} \subseteq\left(D_{\xi}\right)^{y}\right\}$. Then $F_{n} \times Y_{n \xi} \subseteq D_{\xi}$. Also, given $y \in Y,\left(D_{\xi}\right)^{y}(\xi<\mu)$ cover $X$. So, by the definition of a dense family, some $F_{n}$ is contained in some $\left(D_{\xi}\right)^{y}$, i.e., $y \in Y_{n \xi}$.

Corollary. Let $\mathscr{I} \subseteq \mathscr{P}(\mathbb{R})$ be arbitrary. Let $\mu<\kappa$, and let $D_{\xi} \subseteq \mathbb{R} \times \mathbb{R}(\xi<\mu)$ be closed. Suppose that $X \times Y \subseteq \bigcup_{\xi} D_{\xi}$, where $Y \in \mathscr{F}_{\kappa}^{+}$and $X$ has a $\kappa$-dense 
family contained in $\mathscr{F}_{\kappa}^{+}$. Then there exist closed sets $A \in \mathscr{F}_{\kappa}^{+}$and $B \in \mathscr{J}_{\kappa}^{+}$ such that $A \times B \subseteq D_{\xi}$ for some $\xi$.

Proof. Let $\left\{F_{n}: n<\omega\right\} \subseteq \mathscr{F}_{\kappa}^{+}$be $\kappa$-dense for $X$. If in Lemma 1, $Y \in \mathscr{J}_{\kappa}^{+}$, then some $Y_{n \xi} \in \mathscr{J}_{\kappa}^{+}$. Set $A=F_{n}$ and $B=\overline{Y_{n \xi}}$.

Definition. Let $\widetilde{F_{K}}$ be the collection of sets $X \subseteq \bigcup \mathscr{F}$ with the property that for any continuous function $f: Y \rightarrow X, Y \subseteq \mathbb{R}$, there is $W \in \mathscr{T}_{\kappa}$ with $Y \backslash f^{-1}[W] \in \mathscr{M}_{\kappa}$. Note that $\widetilde{\mathscr{F}}_{\kappa}$ is a $\sigma$-ideal, which extends $\mathscr{F}_{\kappa}$.

Lemma 2. If $X \notin \widetilde{\mathscr{F}_{\kappa}}$, then $X$ has a $\kappa$-dense family contained in $\mathscr{F}_{\kappa}^{+}$.

Proof. Suppose $X \notin \widetilde{F}_{\kappa}$. If $X \nsubseteq \cup \mathscr{F}$, then $\{\{x\}\}$ for any $x \in X \backslash \bigcup \mathscr{F}$ is a $\kappa$-dense family. So, let $X \subseteq \bigcup \mathscr{F}$ and let $f: Y \rightarrow X(Y \subseteq \mathbb{R})$ be a continuous function such that $\forall W \in \mathscr{F}_{\kappa} Y \backslash f^{-1}[W] \in \mathscr{M}_{\kappa}^{+}$. Let $\mathscr{U}$ be a countable base for $\mathbb{R}$. Let $V=\bigcup\left\{U \in \mathscr{U}: f[U \cap Y] \in \mathscr{F}_{\kappa}\right\}$. Then $f[V \cap Y] \in \mathscr{T}_{\kappa}$, so, by our assumption, $Y \backslash V \in \mathscr{M}_{\kappa}^{+}$.

As a $\kappa$-dense family we take $\left\{\overline{f[U \cap Y]}: U \in \mathscr{U}\right.$ and $\left.f[U \cap Y] \in \mathscr{F}_{\kappa}^{+}\right\}$. To see that this works let $\mu<\kappa$ and suppose that $X$ is covered by closed sets $D_{\xi} \subseteq \mathbb{R}(\xi<\mu)$. Then the sets $f^{-1}\left[D_{\xi}\right] \cap Y$ cover $Y$ and are relatively closed in $Y$. If $\xi$ is such that for every $U \in \mathscr{U}$ with $U \cap Y \subseteq f^{-1}\left[D_{\xi}\right]$, $f[U \cap Y] \in \mathscr{F}_{\kappa}$, then $f^{-1}\left[D_{\xi}\right] \cap Y \backslash V$ is nowhere dense. Since $Y \backslash V \in \mathscr{M}_{\kappa}^{+}$, this cannot happen to every $\xi$. Thus, we can find $\xi$ and $U$ with $f[U \cap Y] \notin \mathscr{F}_{\kappa}$. Clearly $\overline{f[U \cap Y] \subseteq D_{\xi}}$.

Definition. Say that a sequence $\left\langle F_{\xi}: \xi<\lambda\right\rangle \subseteq \mathscr{F}$ is $\kappa$-cofinal in $\mathscr{F}$ if $\forall F \in$ $\mathscr{F}_{\kappa} \exists \xi<\lambda F \subseteq \bigcup_{\zeta<\xi} F_{\zeta}$.

Lemma 3. Assume $(*)_{\lambda}$. If $\mathscr{F}$ has a $\kappa$-cofinal sequence of length $\lambda$, then $\widetilde{\mathscr{F}}_{\kappa} \subseteq$ $\mathscr{F}_{\lambda}$.

Proof. Fix $X \in \mathscr{F}_{\lambda}^{+}, X \subseteq \cup \mathscr{F}$. Let $\left\langle F_{\xi}: \xi<\lambda\right\rangle \subseteq \mathscr{F}$ be a $\kappa$-cofinal sequence. Pick inductively $x_{\xi} \in X \backslash\left(\left\{x_{\zeta}: \zeta<\xi\right\} \cup \bigcup_{\zeta<\xi} F_{\zeta}\right)(\xi<\lambda)$. This is possible because $X \in \mathscr{F}_{\lambda}^{+}$and $X \subseteq \bigcup \mathscr{F}$. By the construction, $x_{\xi}$ 's are distinct. Also, for every $W \in \mathscr{F}_{\kappa}$ there is $\xi<\lambda$ with $W \subseteq \bigcup_{\zeta<\xi} F_{\zeta}$. Hence $\left|W \cap\left\{x_{\zeta}: \zeta<\lambda\right\}\right|<\lambda$.

By $(*)_{\lambda}$ there is $Y \in \mathscr{M}_{\lambda}^{+}$and a meager-to-one continuous function $f$ : $Y \mapsto\left\{x_{\xi}: \xi<\lambda\right\}$. Note that for every $W \in \mathscr{F}_{\kappa}, f^{-1}[W \cap X] \in \mathscr{M}_{\lambda}$. Since $Y \in \mathscr{M}_{\lambda}^{+}$and $\kappa \leq \lambda$, we have that $Y \backslash f^{-1}[W \cap X] \notin \mathscr{M}_{\kappa}$. Thus $f$ witnesses that $X \notin \widetilde{F_{\kappa}}$.

Corollary. Assume $(*)_{\lambda}$. If $\mathscr{F}$ has a $\kappa$-cofinal sequence of length $\lambda$, then each $X \in \mathscr{F}_{\lambda}^{+}$has a $\kappa$-dense family contained in $\mathscr{F}_{\kappa}^{+}$.

Proof. By Lemmas 2 and 3.

Combining the corollaries of Lemmas 1 and 3 we get the following.

Proposition 1. Let $\kappa \leq \lambda \leq 2^{\aleph_{0}}$ be cardinals, $\operatorname{cf}(\kappa)>\omega$. Assume $(*)_{\lambda}$. Let $\mathscr{I} \subseteq \mathscr{P}(\mathbb{R})$ be arbitrary, and let $\mathscr{F} \subseteq \mathscr{P}(\mathbb{R})$ be a family of closed sets which has a $\kappa$-cofinal sequence of length $\lambda$. Let $\mu<\kappa$ and suppose that $X \times Y \subseteq \bigcup_{\xi} D_{\xi}$, where $D_{\xi} \subseteq \mathbb{R} \times \mathbb{R} \quad(\xi<\mu)$ are closed and $X \in \mathscr{F}_{\lambda}^{+}$and $Y \in \mathscr{I}_{\kappa}^{+}$. 
Then there are closed sets $A \in \mathscr{F}_{\kappa}^{+}$and $B \in \mathscr{F}_{\kappa}^{+}$such that $A \times B \subseteq D_{\xi}$ for some $\xi$.

To make it more transparent that Proposition 1 generalizes the Theorem recall the following notation (see [3]). Let $\mathscr{J} \subseteq \mathscr{P}(\mathbb{R})$ be such that $\bigcup \mathscr{J}=$ $\mathbb{R} \notin \mathcal{J}$.

$$
\begin{aligned}
& \operatorname{cof}(\mathscr{J})=\min \{|\mathscr{A}|: \mathscr{A} \subseteq \mathscr{J}, \forall B \in \mathscr{J} \exists A \in \mathscr{A} B \subseteq A\} \\
& \operatorname{cov}(\mathscr{J})=\min \{|\mathscr{A}|: \mathscr{A} \subseteq \mathscr{J}, \bigcup \mathscr{A}=\mathbb{R}\} \\
& \operatorname{add}(\mathscr{J})=\min \{|\mathscr{A}|: \mathscr{A} \subseteq \mathscr{J}, \bigcup \mathscr{A} \notin \mathscr{J}\} .
\end{aligned}
$$

It is folklore that $\operatorname{add}(\mathscr{J}) \leq \operatorname{cov}(\mathscr{J}) \leq \operatorname{cof}(\mathscr{J})$ and $\operatorname{add}(\mathscr{J}) \leq \operatorname{cf}(\operatorname{cof}(\mathscr{J}))$.

Let now $\mathscr{F}$ be the family of all closed null subsets of $\mathbb{R}$, and $\mathscr{N}$ the $\sigma$-ideal of null subsets of $\mathbb{R}$.

\section{Lemma 4.}

(a) $\operatorname{cov}(\mathscr{M}) \leq \operatorname{cof}(\mathscr{M})$ and $(*)_{\lambda} \Rightarrow \lambda \leq \operatorname{cov}(\mathscr{M}) \quad\left(\right.$ so $(*)_{\operatorname{cof}(\mathscr{M})} \Rightarrow \operatorname{cov}(\mathscr{M})=$ $\operatorname{cof}(\mathscr{M}))$;

(b) $\mathscr{F}_{\operatorname{cov}(\mathscr{M})} \subseteq \mathscr{N}$;

(c) $\operatorname{cof}(\mathscr{F}) \leq \operatorname{cof}(\mathscr{M})$;

(d) $\operatorname{add}(\mathscr{N})$ is a regular cardinal such that $\mathscr{N}_{\operatorname{add}(\mathscr{N})} \subseteq \mathscr{N}$ and $\aleph_{0}<\operatorname{add}(\mathscr{N})$ $\leq \operatorname{add}(\mathscr{M}) \leq \operatorname{cf}(\operatorname{cof}(\mathscr{M})) \leq \operatorname{cof}(\mathscr{M})$.

Proof. (a) is trivial; (d) is well known from Cichoń's diagram (see [3]); (b) is a version of Thm. 2.1 of [7]. We sketch a proof of (c), which is a folklore heir to Thm. 2.1 of [7].

Let $\lambda=\operatorname{cof}(\mathscr{M})$. There exist dominating and diagonalizing families of size $\lambda$, i.e., $\left\{f_{\xi}: \xi<\lambda\right\} \subseteq \omega^{\omega}$ and $\left\{g_{\eta}: \eta<\lambda\right\} \subseteq \omega^{\omega}$ such that

$$
\begin{aligned}
& \forall e \in \omega^{\omega} \exists \xi \forall n e(n)<f_{\xi}(n) ; \\
& \left.\forall h \in \omega^{\omega} \exists \eta \forall n \exists m>n h(m)=g_{\eta}(m)\right)
\end{aligned}
$$

(this is because $\mathfrak{d} \leq \operatorname{cof}(\mathscr{M})$ and $\operatorname{non}(\mathscr{M}) \leq \operatorname{cof}(\mathscr{M})$ in Cichon's diagram; see [3]). We can assume without loss of generality that $\forall \xi \forall n f_{\xi}(n)>n$.

Let $\left\langle I_{i}^{n}: i\langle\omega\rangle\right.$ be an enumeration of all finite unions of closed intervals with rational endpoints with measure $\leq 2^{-n}$. Then the sets

$$
F_{\eta \xi}=\bigcap_{n} \bigcup\left\{I_{g_{\eta}(n)}^{m}: f_{\xi}^{n}(0) \leq m<f_{\xi}^{n+1}(0)\right\}
$$

are closed null and every closed null set is covered by some $F_{\eta \xi}$ (here, $f_{\xi}^{n}=$ $f_{\xi} \circ f_{\xi} \circ \cdots \circ f_{\xi}, n$ times).

Indeed, suppose that $F$ is closed null. Then there is $h \in \omega^{\omega}$ such that $F \subseteq \bigcap_{m} I_{h(m)}^{m}$. Let $\eta$ be such that $\forall n \exists m>n h(m)=g_{\eta}(m)$. Define $e \in \omega^{\omega}$ by $e(n)=\min \left\{m \geq n: h(m)=g_{\eta}(m)\right\}$. Let $\xi$ be such that $\forall n e(n)<f_{\xi}(n)$. Then $\forall n e\left(f_{\xi}^{n}(0)\right)<f_{\xi}^{n+1}(0)$, so $\forall n \exists m \in\left[f_{\xi}^{n}(0), f_{\xi}^{n+1}(0)\right) h(m)=g_{\eta}(m)$. It follows that $\bigcap_{m} I_{h(m)}^{m} \subseteq F_{\eta \xi}$.

From Proposition 1 we get the following.

Proposition 2. Assume $(*)_{\operatorname{cof}(\mathscr{A})}$. If less than $\operatorname{add}(\mathscr{N})$ closed subsets of $\mathbb{R} \times \mathbb{R}$ cover a nonnull rectangle $X \times Y$, then some of them cover a closed nonnull rectangle $A \times B$. 
Proof. Use Lemma 4. Let $\lambda=\operatorname{cof}(\mathscr{M}), \kappa=\operatorname{add}(\mathscr{N})$. By $(\mathrm{d}), \aleph_{0}<\operatorname{cf}(\kappa)$ and $\kappa \leq \lambda$. By $(\mathrm{a}), \lambda=\operatorname{cov}(\mathscr{M})$. So, by (b), $X \in \mathscr{F}_{\lambda}^{+}$; and by $\mathscr{N}_{\kappa} \subseteq \mathscr{N}, Y \in \mathscr{N}_{\kappa}^{+}$. Also by $(\mathrm{c})$ and $\kappa \leq \operatorname{cf}(\lambda), \mathscr{F}$ has a $\kappa$-cofinal sequence of length $\lambda$. Now use Proposition 1 for $\mathscr{I}=\mathscr{N}$.

Note that by Lemma $4\left(\right.$ a),$(*)_{2^{\aleph_{0}}} \Rightarrow \operatorname{cov}(\mathscr{M})=\operatorname{cof}(\mathscr{M})=2^{\aleph_{0}}$. So, Proposition 2 directly generalizes the Theorem.

We conclude the paper with the following variant of Proposition 2.

Proposition 3. Assume $(*)_{\operatorname{cof}(\mathscr{M})}$. Suppose that a coanalytic set $C \subseteq \mathbb{R} \times \mathbb{R}$, whose all horizontal sections are unions of less than $\operatorname{cf}(\operatorname{cof}(\mathscr{M}))$ closed sets, contains a nonnull rectangle $X \times Y$. Then it contains a closed nonnull rectangle $A \times B$.

Proof. Let $\lambda=\operatorname{cof}(\mathscr{M}), \kappa=\operatorname{cf}(\lambda)$. As in the proof of Proposition 2 we get that $X \in \mathscr{F}^{+}$and that there is a $\kappa$-cofinal sequence for $\mathscr{F}$ of length $\lambda$. So, by the corollary to Lemma $3, X$ has a $\kappa$-dense family $\left\{F_{n}: n<\omega\right\} \subseteq \mathscr{F}_{\kappa}{ }^{+}$.

Let $G_{n}=\left\{y: F_{n} \subseteq C^{y}\right\}$. Note that $G_{n}$ 's are analytic. Also, they cover $Y\left(C^{y}\right.$ is a union of less than $\kappa$ closed sets, so, by $\kappa$-density, $C^{y}$ contains some $F_{n}$ ). As $Y$ is nonnull, some $G_{n}$ is nonnull and, hence, being measurable, contains a closed nonnull subset. Since $F_{n}$ is also closed nonnull and $F_{n} \times G_{n} \subseteq$ $C$, we are done.

Note. Our rectangles had sides parallel to the coordinate axes. What if we consider arbitrary rectangles. The sets $E$ and $F$ discussed at the beginning of the paper have a stronger property than stated. Namely, if $E$ contains a rectangle $R$ with one side nonnull and the other measurable and nonnull, then one of the sides must be parallel to the line $y=-x$. Indeed, otherwise $R$ is obtained by a rotation by an angle $\alpha, 0 \leq \alpha<\pi / 4$, of a rectangle $X \times Y$. So, $R=\left\{\left\langle x^{*}, y^{*}\right\rangle: x \in X, y \in Y\right\}$, where $x^{*}=x \cos \alpha-y \sin \alpha$ and $y^{*}=x \sin \alpha+y \cos \alpha$. From $R \subseteq E$ we get that for all $x \in X$ and $y \in Y$, $x^{*}+y^{*} \in F$, i.e., $x(\cos \alpha+\sin \alpha)+y(\cos \alpha-\sin \alpha) \in F$. Thus, $a \cdot X+b \cdot Y \subseteq F$ for some nonzero $a$ and $b$. As before, if one of $X, Y$ is nonnull and the other is measurable and nonnull, we get a contradiction.

Now let $E^{\prime}$ be a rotation of $E$ by an angle $\beta, 0<\beta<\pi$. Then $E \cap E^{\prime}$ is a conull subset of the Euclidean plane, which contains no rectangle with one side nonnull and the other measurable and nonnull. Thus, from the following weaker version of $(+)$ :

$(+')$ if an $\mathbf{F}_{\sigma}$ subset of $\mathbb{R} \times \mathbb{R}$ contains a nonnull rectangle, then it contains a measurable nonnull rectangle;

we can construct a conull subset of the plane which contains no nonnull rectangle.

\section{REFERENCES}

1. M. Burke, A theorem of Friedman on rectangle inclusion and its consequences, Note of March 7, 1991.

2. T. Bartoszyński, M. Goldstern, H. Judah, and S. Shelah, All meager filters may be null, Proc. Amer. Math. Soc. 117 (1993), 515-521.

3. T. Bartoszyński and $\mathrm{H}$. Judah, Measure and category in set theory (forthcoming book).

4. H. G. Eggleston, Two measure properties of cartesian product sets, Quart. J. Math. Oxford (2) 5 (1954), 108-115. 
5. D. H. Fremlin, Problem list, circulated notes, 1987.

6. D. H. Fremlin and M. Talagrand, $A$ decomposition theorem for additive set functions and applications to Pettis integral and ergodic means, Math. Z. 168 (1979), 117-142.

7. A. Miller, Some properties of measure and category, Trans. Amer. Math. Soc. 266 (1981), 93-114.

8. __ Mapping a set of reals onto the reals, J. Symbolic Logic 48 (1983), 575-584.

9. J. Oxtoby, Measure and category, Springer-Verlag, Berlin, 1970.

Department of Mathematics, University of Wroclaw, Pl. Grunwaldzki 2/4, 50-156 Wroclaw, Poland

E-mail address: pawlikow@math.uni.wroc.pl 